\title{
Dynamical Supersymmetric Inflation
}

\author{
William H. Kinney* and Antonio Riotto ${ }^{\dagger}$ \\ NASA/Fermilab Astrophysics Center \\ Fermi National Accelerator Laboratory, Batavia, IL 60510 \\ FERMILAB-PUB-97/090-A \\ hep-ph/9704388
}

(April 23, 1997)

\begin{abstract}
We propose a new class of inflationary models in which the scalar field potential governing inflation is generated by the same non-perturbative gauge dynamics that may lead to supersymmetry breaking. Such models satisfy constraints from cosmic microwave background measurements for natural values of the fundamental parameters in the theory. In addition, they have two particularly interesting characteristics: a "blue" spectrum of scalar perturbations, and an upper bound on the total amount of inflation possible.

98.80.Cq,12.60.Jv
\end{abstract}

Typeset using REVTEX

*Electronic mail: kinneyw@fnal.gov

${ }^{\dagger}$ EPPARC Advanced Fellow, Oxford Univ. from Sept. 1997. From 1 Dec. 1997 on leave of absence at CERN, Theory Division, CH-1211 Geneva 23, Switzerland. Electronic mail: riotto@fnas01.fnal.gov. 


\section{INTRODUCTION}

The existence of an inflationary stage during the evolution of the early Universe is usually invoked to solve the flatness and the horizon problems of the standard big bang cosmology [1]. During inflation the energy density is dominated by vacuum energy and comoving scales undergo quasi-exponential growth. As a result, any undesirable topological defects left as remnants after some Grand Unified phase transition, such as monopoles, are diluted. Typically, the vacuum energy driving inflation is generated by a scalar field $\phi$ (the "inflaton") displaced from the minimum of a potential $V(\phi)$. Quantum fluctuations of the inflaton field imprint a nearly scale invariant spectrum of fluctuations on the background space-time metric. These fluctuations may be responsible for the generation of structure formation. However, the level of density and temperature fluctuations observed in the present Universe, $\delta \rho / \rho \sim 10^{-5}$, require the inflaton potential to be extremely flat. For instance, in the chaotic inflationary scenario [2] where the inflaton potential is $V=\lambda \phi^{4}$ and the scalar field sits initially at scales of order of the Planck scale, the dimensionless self-coupling $\lambda$ must be of order of $10^{-13}$ to be consistent with observations. The inflaton field must be coupled to other fields in order to ensure the conversion of the vacuum energy into radiation at the end of inflation, but these couplings must be very small, otherwise loop corrections to the inflaton potential spoil its flatness. While the necessity of introducing very small parameters to ensure the extreme flatness of the inflaton potential seems very unnatural and fine-tuned in most non-supersymmetric theories, this technical naturalness may be achieved in supersymmetric models [3] because the nonrenormalization theorem guarantees that the superpotential is not renormalized to all orders of perturbation theory 仙. However, even though the form of the potential may be stable when the inflaton couples to other fields, initial small couplings $\lambda$ must still be put by hand, an aesthetically unpleasant option. A natural way to solve this problem is to interpret small couplings as a ratio of different mass scales. This is the underlying idea of hybrid inflation [5, 6] where two (or more) interacting fields are associated with different scales and inflation ends by a rapid rolling of a sec- 
ond field $\sigma$, initially sitting at the origin and triggered by the slow rolling of the field $\phi$. During the inflationary stage the inflaton potential is typically given by $V=V_{0}+\frac{m^{2}}{2} \phi^{2}$ and the smallness of the density perturbations may be easily explained by the hierarchy $m<V_{0}^{1 / 4} \ll M_{P l}$. Successful supersymmetric versions of hybrid inflation have been constructed in the framework of global supersymmetry [7] and, more recently, in supergravity theories [8]. Supersymmetry can therefore play a fundamental role during inflation [9].

In our discussion of inflation below we will concern ourselves with a potential of the form:

$$
V(\phi)=\frac{\Lambda_{3}^{p+4}}{\phi^{p}},
$$

where the index $p$ and the scale $\Lambda_{3}$ depend upon the underlying gauge group. Such a potential might at first seem peculiar since it involves a field (or more than one field) appearing in the denominator. However, such potentials are known to arise generically in supersymmetric theories 1 . This fact has been known for some time [10], and has gained increasing attention of late after the recent work on understanding the non-perturbative behavior of supersymmetric gauge theories [11]. The unconventional term involving fields in the denominator typically arises due to non-perturbative effects that lift various flat directions in supersymmetric theories. Such non-perturbative effects might have to do with instanton effects or, for example, with gaugino condensation associated with some unbroken non-Abelian gauge group. It is also worth mentioning that regardless of exactly how the potential in Eq. (1) arises, in our discussion of inflation we will be working in the region where the field(s) involved have large enough vacuum expectation values that their presence in the denominator does not cause any singular behavior. In this regime the theory is weakly coupled and corrections to the canonical Kahler potential are negligible.

\footnotetext{
${ }^{1}$ Strictly speaking we are referring here to the superpotential in a supersymmetric theory. When the superpotential arises due to instanton effects, and when the gauge groups involved are completely broken at a high enough scale, one can argue that the resulting potential has a similar form.
} 
We will not be concerned, in this paper, with the the detailed dynamics which gives rise to the potential in Eq. (1). This is in large part because our conclusions are quite insensitive to most details of the potential and follow mainly from the general form of Eq. (1). It is of course interesting to ask whether the inflationary scenario envisaged in this paper can be actually realized in a reasonably simple supersymmetric model. It has been suggested, for example in models of gauge mediated supersymmetry breaking, that non-perturbative effects, driven by instanton effects or gaugino condensation, might be responsible for the dynamical supersymmetry breaking (DSB) when the classical moduli space is lifted in the potential and scalar fields are driven to large expectation values. Additional tree level interactions in the potential may raise the potential at large expectation values leading to a stable ground state. If some $F$-terms (and the potential) do not vanish in such a ground state, supersymmetry is spontaneously broken. Recent developments have also shown that many supersymmetric theories may have other types of non-perturbative dynamics which lead to degenerate quantum moduli spaces of vacuum instead of dynamically generated superpotentials.

This makes it tempting to speculate (although it is not necessary for the discussion of inflation proposed in this paper) that the potential above, responsible for inflation, could also arise from the same underlying dynamics responsible for supersymmetry breaking. We will not pursue these ideas any further here and leave them for future study. We only note in passing that the term in Eq. (11) is not usually the only one present in the potential. Other terms may be present and lift up the flat direction. If these terms are small enough, the vacuum expectation value of the field is finite, but nevertheless all the interesting dynamics of inflation happens when the term (1) dominates. This comes out naturally if these other terms are nonrenormalizable, which is plausible in many DSB models. Moreover, following the philosophy of such models, it is likely that nonrenormalizable terms are suppressed by powers of $M_{P l}$, the only explicit scale allowed in the theory.

The last key ingredient of our proposal is that during inflation, the energy density is dominated by a a nonzero constant vacuum energy density $V_{0}$ (this always happens if the 
value of the inflaton field is large enough). In the absence of any other physics, the inflaton field rolls down to its VEV and inflation never ends. However, the inflaton may be coupled to some fields in some other sector of the theory in such away that, when $\phi$ gets larger than some critical value $\phi_{c}, V_{0}$ drops to zero. This is the usual way inflation is terminated in hybrid inflation. What is remarkable is that this vacuum energy density $V_{0}$ may be identified with some scale $\Lambda_{2}^{4}$ which appears in models where quantum deformation of a classical moduli space plays a fundamental role. A typical example is provided by the chiral $S U(2) \otimes S U(3)$ model in the limit in which the scale associated to $S U(2)$ is much higher than the one associated to $S U(3)$. Here we are assuming that the scale $V_{0}$ is only present during the inflationary stage and relaxes to zero to opportunely end inflation.

In this paper we propose a new class of inflationary models inspired by the typical structure of the potential generated by some non-perturbative gauge dynamics. We will denote this class of models by dynamical supersymmetric inflation (DSI). We will show that a successful inflationary scenario may be constructed and that the generation of density perturbations may be accounted for in the limit $\Lambda_{2} \gg \Lambda_{3}$. More interestingly, a blue spectrum of density perturbations is predicted.

The paper is organized as follows: Section (피) is a short review of inflationary cosmology from scalar field theories. Section (III) contains the details of limiting the parameters of the model from observations of CMB fluctuations. Finally, Section (IV) contains a summary and conclusions.

\section{INFLATION IN SCALAR FIELD THEORIES}

In this section, we quickly review scalar field models of inflationary cosmology, and explain how we relate model parameters to observable quantities. (For more detailed reviews, see Refs. [12,9].) If the stress-energy of the universe is dominated by a scalar field with potential $V(\phi)$, the Einstein Field equations $G_{\mu \nu}=\left(8 \pi / M_{P l}^{2}\right) T_{\mu \nu}$ for the evolution of the background metric reduce to 


$$
H^{2} \equiv\left(\frac{\dot{a}}{a}\right)^{2}=\frac{8 \pi}{3 M_{P l}^{2}}\left[\frac{1}{2} \dot{\phi}^{2}+V(\phi)\right]
$$

Here $a(t)$ is the scale factor, and $M_{P l}=G^{-1 / 2} \simeq 10^{19} \mathrm{GeV}$ is the Planck mass. Inflation is defined to be a period of accelerated expansion, $\ddot{a}>0$. The evolution of the scale factor can be written as $a \propto e^{N}$, where the number of e-folds $N$ is defined in terms of the Hubble parameter $H$ as

$$
N \equiv \int H d t
$$

During inflation $H$, and therefore the horizon size $d_{H} \simeq H^{-1}$, is nearly constant, and the expansion of the universe is quasi-exponential. This results in the curious behavior that the coordinate system is expanding faster than the light traveling in it, and comoving length scales rapidly increase in size relative to the horizon distance. Regions initially in causal contact are "redshifted" to large, non-causal scales, explaining the observed isotropy of the cosmic microwave background (CMB) on large angular scales. This is also important for the generation of metric fluctuations in inflation, discussed below. Finally, a universe which starts out with a nonzero curvature evolves rapidly during inflation toward zero curvature and a flat Robertson-Walker metric.

Stress-energy conservation gives the equation of motion of the scalar field

$$
\ddot{\phi}+3 H \dot{\phi}+V^{\prime}(\phi)=0
$$

The slow-roll approximation [13,14 is the assumption that the evolution of the field is dominated by drag from the cosmological expansion, so that $\ddot{\phi} \simeq 0$ and

$$
\dot{\phi} \simeq-\frac{V^{\prime}}{3 H}
$$

The equation of state of the scalar field is then dominated by the potential, so that $p \simeq-\rho$, and the expansion rate is approximately

$$
H \simeq \sqrt{\frac{8 \pi}{3 M_{P l}^{2}} V(\phi)} .
$$


The slow-roll approximation is consistent if both the slope and curvature of the potential are small. This condition is conventionally expressed in terms of the "slow-roll parameters" $\epsilon$ and $\eta$, where

$$
\epsilon \equiv \frac{M_{P l}^{2}}{4 \pi}\left(\frac{H^{\prime}(\phi)}{H(\phi)}\right) \simeq \frac{M_{P l}^{2}}{16 \pi}\left(\frac{V^{\prime}(\phi)}{V(\phi)}\right)^{2}
$$

and

$$
\eta(\phi) \equiv \frac{M_{P l}^{2}}{4 \pi}\left(\frac{H^{\prime \prime}(\phi)}{H(\phi)}\right) \simeq \frac{M_{P l}^{2}}{8 \pi}\left[\frac{V^{\prime \prime}(\phi)}{V(\phi)}-\frac{1}{2}\left(\frac{V^{\prime}(\phi)}{V(\phi)}\right)^{2}\right] .
$$

Slow-roll is then a consistent approximation for $\epsilon, \eta \ll 1$. The parameter $\epsilon$ can be shown to directly parameterize the equation of state of the scalar field, $p=-\rho(1-2 / 3 \epsilon)$, so that the condition for inflation $\ddot{a}>0$ is exactly equivalent to $\epsilon<1$. The number of e-folds $N$ of inflation as the field evolves from $\phi_{i}$ to $\phi_{f}$ can be expressed in terms of $\epsilon$ as

$$
N=\frac{2 \sqrt{\pi}}{M_{P l}} \int_{\phi_{i}}^{\phi_{f}} \frac{d \phi}{\sqrt{\epsilon(\phi)}} .
$$

To match the observed degree of flatness and homogeneity in the universe, we require many e-folds of inflation, typically $N \simeq 50$. (This figure varies somewhat with the details of the model.)

Inflation not only explains the high degree of large-scale homogeneity in the universe, but also provides a mechanism for explaining the observed inhomogeneity as well. During inflation, quantum fluctuations on small scales are quickly redshifted to scales much larger than the horizon size, where they are "frozen" as perturbations in the background metric [15 18. Metric perturbations at the surface of last scattering are observable as temperature anisotropy in the CMB, which was first detected by the Cosmic Background Explorer (COBE) satellite. The metric perturbations created during inflation are of two types: scalar, or curvature perturbations, which couple to the stress-energy of matter in the universe and form the "seeds" for structure formation, and tensor, or gravitational wave perturbations, which do not couple to matter. Both scalar and tensor perturbations contribute to CMB anisotropy. Scalar fluctuations can also be interpreted as fluctuations in the density of the 
matter in the universe. Scalar fluctuations can be quantitatively characterized by perturbations $P_{\mathcal{R}}$ in the intrinsic curvature [19 22]

$$
P_{\mathcal{R}}^{1 / 2}(k)=\left.\frac{1}{\sqrt{\pi}} \frac{H}{M_{P l} \sqrt{\epsilon}}\right|_{k^{-1}=d_{H}} .
$$

The fluctuation power is in general a function of wavenumber $k$, and is evaluated when a given mode crosses outside the horizon during inflation, $k^{-1}=d_{H}$. Outside the horizon, modes do not evolve, so the amplitude of the mode when it crosses back inside the horizon during a later radiation or matter dominated epoch is just its value when it left the horizon during inflation. The spectral index $n_{\mathcal{R}}$ is defined by assuming an approximately power-law form for $P_{\mathcal{R}}$ with

$$
n_{\mathcal{R}}-1 \equiv \frac{d \ln \left(P_{\mathcal{R}}\right)}{d \ln (k)},
$$

so that a scale-invariant spectrum, in which modes have constant amplitude at horizon crossing, is characterized by $n_{\mathcal{R}}=1$. Instead of specifying the fluctuation amplitude directly as a function of $k$, it is often convenient to specify it as a function of the number of e-folds $N$ before the end of inflation at which a mode crossed outside the horizon. Scales of interest for measurements of $\mathrm{CMB}$ anisotropy crossed outside the horizon at $N \simeq 50$, so that $P_{\mathcal{R}}$ is conventionally evaluated at $P_{\mathcal{R}}(N=50)$. Similarly, the power spectrum of tensor fluctuation modes is given by

$$
P_{T}^{1 / 2}\left(k_{N}\right)=\left.\frac{4}{\sqrt{\pi}} \frac{H}{M_{P l}}\right|_{N=50} .
$$

The ratio of tensor to scalar modes is $\left(P_{T} / P_{\mathcal{R}}\right)=16 \epsilon$, so that tensor modes are negligible for $\epsilon \ll 1$. If the contribution of tensor modes to the CMB anisotropy can be neglected, normalization to the COBE four-year data gives [23,9] $P_{\mathcal{R}}^{1 / 2}=5 \times 10^{-5}$. Calculating the CMB fluctuations from a particular inflationary model reduces to the following basic steps: (1) from the potential, calculate $\epsilon$ and $\eta$. (2) From $\epsilon$, calculate $N$ as a function of the field $\phi$. (3) Invert $N(\phi)$ to find $\phi_{N=50}$. (4) Calculate $P_{\mathcal{R}}, n_{\mathcal{R}}$, and $P_{T}$ as functions of $\phi$, and evaluate at $\phi_{N=50}$ to determine the values of the observables at scales of current astrophysical interest. 


\section{INFLATION FROM NON-PERTURBATIVE GAUGE DYNAMICS IN SUPERSYMMETRY}

We take the potential to be described by a single degree of freedom $\phi$, of the general form

$$
V(\phi)=V_{0}+\frac{\Lambda_{3}^{p+4}}{\phi^{p}}+\frac{\phi^{q+4}}{M_{P l}^{q}} .
$$

As we noted in the introduction, the presence of the nonrenormalizable term is not strictly necessary. We include it here for generality, and show that its presence, subject to certain consistency constraints, does not significantly affect our conclusions.

The minimum of the potential is at $V^{\prime}(\langle\phi\rangle)=0$, where the vacuum expectation value $(\mathrm{VEV})\langle\phi\rangle$ is given by

$$
\langle\phi\rangle=\left[\left(\frac{p}{q+4}\right) \Lambda_{3}^{p+4} M_{P l}^{q}\right]^{1 /(p+q+4)} .
$$

Note that, in general, the potential does not vanish when the field is at the VEV. This means that if the Universe ever becomes vacuum dominated, it stays vacuum dominated, and inflation will continue indefinitely unless other physics is brought into play. Here we will simply assume that some other sector of the theory ends inflation when $\phi$ passes through a critical value $\phi_{c}$. For $\phi_{c}$ near the $\operatorname{VEV}\langle\phi\rangle$, the potential can be expanded as

$$
V(\phi)=V(\langle\phi\rangle)+\frac{1}{2} V^{\prime \prime}(\langle\phi\rangle)(\phi-\langle\phi\rangle)^{2}+\cdots
$$

This is just the case of standard hybrid inflation [5,6], which has been studied extensively in

the literature. Here we study the limit $\phi \ll\langle\phi\rangle$, for which such an expansion is not possible. In this limit, the $\phi^{-p}$ term dominates the dynamics,

$$
\begin{aligned}
V(\phi) & \simeq V_{0}+\frac{\Lambda_{3}^{p+4}}{\phi^{p}}, \quad \phi \ll\langle\phi\rangle \\
& =V_{0}\left[1+\alpha\left(\frac{M_{P l}}{\phi}\right)^{p}\right],
\end{aligned}
$$

where 


$$
\alpha \equiv \frac{\Lambda_{3}^{p+4}}{M_{P l}^{p} V_{0}} .
$$

We assume that the constant $V_{0}$ dominates the potential, or $\alpha \ll\left(\phi / M_{P l}\right)^{p}$. In this limit, the first slow-roll parameter is

$$
\epsilon(\phi)=\frac{M_{P l}^{2}}{16 \pi}\left(\frac{V^{\prime}(\phi)}{V(\phi)}\right)^{2}=\left(\frac{\phi_{0}}{\phi}\right)^{2(p+1)},
$$

where

$$
\left(\frac{\phi_{0}}{M_{P l}}\right)=\left(\frac{p}{4 \sqrt{\pi}} \alpha\right)^{1 /(p+1)} .
$$

The second slow-roll parameter $\eta$ is

$$
\begin{aligned}
\eta(\phi) & =\epsilon-\frac{M_{P l}}{4 \sqrt{\pi}} \frac{\epsilon^{\prime}}{\sqrt{\epsilon}} \\
& =\left(\frac{\phi_{0}}{\phi}\right)^{2(p+1)}+\left(\frac{p+1}{2 \sqrt{\pi}}\right)\left(\frac{\phi_{0}}{\phi}\right)^{p+1}\left(\frac{M_{P l}}{\phi}\right) .
\end{aligned}
$$

Note that for $\phi \simeq \phi_{0} \ll M_{P l}$, the parameter $\eta$ becomes large, indicating a breakdown of the slow-roll approximation. In particular, it is inconsistent to say that inflation begins at $\phi=\phi_{0}$, when $\epsilon(\phi)$ in Eq. (18) is equal to unity, since that expression depends on the assumption of slow-roll. However, for $\phi \gg \phi_{0}$, both $\epsilon$ and $\eta$ are small and slow-roll is a consistent approximation. In the region $\phi_{0} \ll \phi \ll M_{P l}$, the second term in (20) dominates, which is equivalent to $\eta \gg \epsilon$, and $\eta$ can be written in the useful forms

$$
\begin{aligned}
\eta(\phi) & \simeq \frac{p+1}{2 \sqrt{\pi}} \sqrt{\epsilon(\phi)}\left(\frac{M_{P l}}{\phi}\right) \\
& =\frac{p(p+1)}{8 \pi} \alpha\left(\frac{M_{P l}}{\phi}\right)^{p+2} .
\end{aligned}
$$

The number of e-folds $N$ is given by

$$
\begin{aligned}
N=\frac{2 \sqrt{\pi}}{M_{P l}} \int_{\phi}^{\phi_{c}} \frac{d \phi^{\prime}}{\sqrt{\epsilon\left(\phi^{\prime}\right)}} & =\left.\left(\frac{p+1}{p+2}\right) \frac{1}{\eta-\epsilon}\right|_{\phi} ^{\phi_{c}} \\
& \simeq\left(\frac{p+1}{p+2}\right)\left(\frac{1}{\eta\left(\phi_{c}\right)}-\frac{1}{\eta(\phi)}\right), \quad \epsilon \ll \eta,
\end{aligned}
$$

where $\phi_{c}$ is the critical value at which inflation ends. The value of $\phi_{c}$ is in general determined by a coupling of the field $\phi$ to some other sector of the theory which we have here left 
unspecified. Accordingly, we will treat $\phi_{c}$ as simply a free parameter. Noting from Eq. (21) that $\eta \propto \phi^{-(p+2)}$, for $\phi \ll \phi_{c}$ the number of e-folds $N$ approaches a constant, which we call $N_{\text {tot }}$,

$$
N_{\text {tot }} \equiv\left(\frac{p+1}{p+2}\right) \frac{1}{\eta\left(\phi_{c}\right)}=\frac{8 \pi}{p(p+2)} \alpha^{-1}\left(\frac{\phi_{c}}{M_{P l}}\right)^{p+2}
$$

This is quite an unusual feature. Most models of inflation have no intrinsic upper limit on the total amount of expansion that takes place during the inflationary phase, although only the last 50 or 60 e-folds are of direct observational significance. Here the total amount of inflation is bounded from above, although that upper bound can in principle be very large. Defining $\phi_{N}$ to be the field value $N$ e-folds before the end of inflation, we can then write $\eta\left(\phi_{N}\right)$ in terms of $N$ and $N_{\text {tot }}$ as

$$
\eta\left(\phi_{N}\right)=\left(\frac{p+1}{p+2}\right) \frac{1}{N_{\text {tot }}-N}
$$

so that $\eta$ approaches a constant value for $N \ll N_{\text {tot }}$. The magnitude of scalar metric perturbations is given by the curvature power spectrum $P_{\mathcal{R}}$,

$$
\begin{aligned}
P_{\mathcal{R}}^{1 / 2} & \equiv \frac{1}{\sqrt{\pi}} \frac{H\left(\phi_{50}\right)}{M_{P l} \sqrt{\epsilon\left(\phi_{50}\right)}} \\
& =\frac{(p+1)}{\pi} \sqrt{\frac{2 \pi}{3}}\left(\frac{V_{0}^{1 / 2}}{M_{P l} \phi_{50}}\right) \frac{1}{\eta\left(\phi_{50}\right)} \\
& =\frac{(p+2)}{\pi} \sqrt{\frac{2 \pi}{3}}\left(\frac{V_{0}^{1 / 2}}{M_{P l} \phi_{c}}\right) N_{\mathrm{tot}}\left(1-\frac{50}{N_{\mathrm{tot}}}\right)^{(p+1) /(p+2)}
\end{aligned}
$$

The COBE normalization is $23,9 P_{\mathcal{R}}^{1 / 2}=5 \times 10^{-5}$, with spectral index

$$
\begin{aligned}
n_{\mathcal{R}}-1 & \equiv \frac{d \log \left(P_{\mathcal{R}}\right)}{d \log (k)}=-4 \epsilon+2 \eta \\
& \simeq\left(\frac{p+1}{p+2}\right) \frac{2}{N_{\text {tot }}\left(1-50 / N_{\text {tot }}\right)} .
\end{aligned}
$$

As announced, the spectrum turns out to be blue, but for $N_{\text {tot }} \gg 50$ the spectrum approaches scale-invariance, $n_{\mathcal{R}} \simeq 1$. If we take the example case of $p=2$ and $\phi_{c} \sim V_{0}^{1 / 4}$, the COBE constraint on $P_{\mathcal{R}}$ is met for $V_{0}^{1 / 4} \simeq 10^{10} \mathrm{GeV}$ and $\Lambda_{3} \simeq 10^{6} \mathrm{GeV}$, very natural values for 
the fundamental scales in the theory. Since $V_{0}^{1 / 4} \ll M_{P l}$, tensor modes produced during inflation are of negligible amplitude, a typical feature of hybrid inflation models.

In this class of models, a nearly scale-invariant spectrum $n_{\mathcal{R}} \simeq 1$ is the most natural case. Significantly blue spectra, $n_{\mathcal{R}}>1$, are, however, not excluded and can occur for properly tuned values of the fundamental scales in the theory. The condition for significant deviation from a scale-invariant spectrum is that $N_{\text {tot }}$ not be much greater than 50 . We can write $N_{\text {tot }}$ as a function of the scalar spectral index

$$
N_{\text {tot }}=50+\left(\frac{p+1}{p+2}\right) \frac{2}{n_{\mathcal{R}}-1},
$$

so, again taking $p=2$, a spectral index of $n_{\mathcal{R}}>1.1$ requires $N_{\text {tot }}<65$. Such a small amount of inflation could have observationally important consequences [24].

We have two observationally determined constraints, $P_{\mathcal{R}}^{1 / 2}=5 \times 10^{-5}$ and $n_{\mathcal{R}}<1.5$, and three free parameters in the model, $\phi_{c}, \Lambda_{3}$, and $\Lambda_{2} \equiv V_{0}^{1 / 4}$. Then fixing the spectral index $n_{\mathcal{R}}$ and varying $\phi_{c}$ results in a contour in the $\Lambda_{2} / \Lambda_{3}$ plane. In addition, we have two consistency constraints: first that the dynamics of the field are dominated by the $\phi^{-p}$ term, which is equivalent to the condition

$$
\phi<\phi_{c} \ll\langle\phi\rangle=\left[\left(\frac{p}{q+4}\right) \Lambda_{3}^{p+4} M_{P l}^{q}\right]^{1 /(p+q+4)},
$$

and second that the vacuum energy is dominated by the constant $V_{0} \equiv \Lambda_{2}^{4}$, which can be expressed as

$$
\phi \gg \alpha^{1 / p} M_{P l}=\left(\frac{\Lambda_{3}^{p+4}}{\Lambda_{2}^{4}}\right)^{1 / p}
$$

Fig 1. shows constant $n_{\mathcal{R}}$ contours in the $\Lambda_{2} / \Lambda_{3}$ plane for an ensemble of distinct cases. The interesting feature of this plot is that the COBE limits on the fundamental parameters of the model are relatively insensitive to the choice of $p$ and $q$, and hence on the details of the underlying physical theory. Further, if future observations find a spectrum of density fluctuations which detectably deviates from the $n_{\mathcal{R}}=1$ scale-invariant case, this will significantly constrain the range of allowed parameters. Of particular note is that all of the 
fundamental mass scales are significantly below the Planck scale, $\Lambda_{2}, \Lambda_{3} \ll M_{P l}$. Finally, it is interesting to note that the presence of nonrenormalizable terms in the Lagrangian does not significantly affect our conclusions, so that the scenario is quite robust.

\section{CONCLUSIONS}

A generic feature of models of nonperturbative gauge dynamics in supersymmetry is the presence of a "scalar field" potential of the form

$$
V(\phi)=\frac{\Lambda_{3}^{p+4}}{\phi^{p}},
$$

where the field $\phi$ is in general a label for a condensate. In this paper, we consider this general form of a scalar field potential in the context of inflationary cosmology, and find that an inflationary phase in the very early universe is a generic and natural characteristic, for example, of dynamical supersymmetry breaking. We call this class of inflationary models "Dynamical Supersymmetric Inflation (DSI)." Like models of hybrid inflation, these models are characterized by a potential dominated by a constant term $V_{0}$, and require coupling to another sector to end inflation when $\phi$ reaches a critical value $\phi_{c}$. Unlike standard hybrid inflation models, models of this type postulate a field far from the minimum of the potential, $\phi_{c} \ll\langle\phi\rangle$.

The primary observational constraint on models of inflation comes from the Cosmic Background Explorer (COBE) satellite, which measured fluctuations in the cosmic microwave background of magnitude $P_{\mathcal{R}}=5 \times 10^{-5}$, with a spectral index $n_{\mathcal{R}}=1.2 \pm 0.3$. The small fluctuation amplitude, which requires fine-tuned dimensionless parameters in typical infla-

tion models, appears naturally here as a ratio of fundamental scales, e.g. $V_{0}^{1 / 4} \simeq 10^{10} \mathrm{GeV}$ and $\Lambda_{3} \simeq 10^{6} \mathrm{GeV}$. We do not pursue here the idea that the potential responsible for inflation could also arise from the same underlying dynamics responsible for supersymmetry breaking. It is, however, intriguing to notice that the scales $\Lambda_{2}$ and $\Lambda_{3}$ turn out to be of the right order of magnitude to explain supersymmetric particle masses in the TeV range in the supergravity and gauge mediated supersymmetry breaking scenarios, respectively. 
DSI is characterized by a "blue" spectral index, $n_{\mathcal{R}}>1$. A nearly scale-invariant spectrum $n_{\mathcal{R}} \simeq 1$ is the most natural outcome, but significantly blue spectra can occur for reasonable values of the parameters. The addition of nonrenormalizable terms suppressed by powers of the Planck mass,

$$
V=\frac{\phi^{q+4}}{M_{P l}^{q}},
$$

does not significantly alter the inflationary properties of the potential.

Models of cosmological inflation based on dynamical supersymmetry breaking are not only well motivated from a particle physics standpoint, but also very naturally meet constraints from observations of the CMB. These models have the unusual characteristic of possessing an upper limit on the total amount of inflation, as well as the attractive feature of predicting a "blue" spectrum of density fluctuations.

\section{ACKNOWLEDGMENTS}

This work was supported in part by DOE and NASA grant NAG5-2788 at Fermilab. We would like to thank Sandip Trivedi at Fermilab for a number of helpful discussions and for participating to the early stages of this work. 


\section{FIGURES}

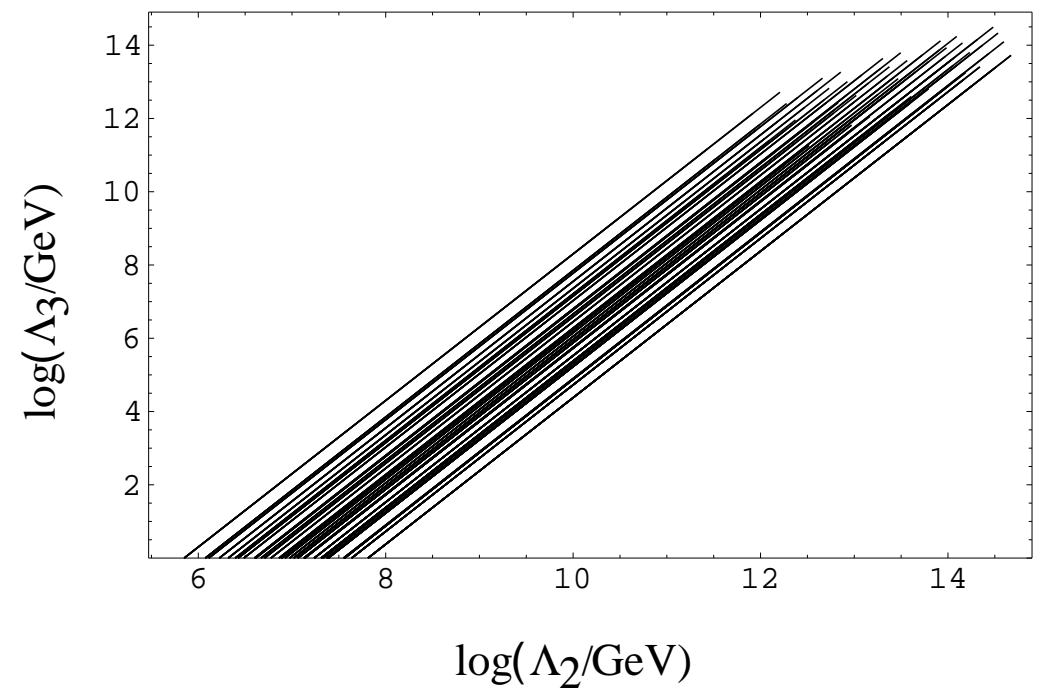

Fig. 1: Constant $n_{R}$ contours in the $\Lambda_{2}-\Lambda_{3}$ plane for two hundred cases with $p$ and $q$ varying from 2 to 10 and the scalar spectral index varying from $n_{\mathcal{R}}=1.0001$ to $n_{\mathcal{R}}=1.5$. Note that all the cases result in similar limits on $\Lambda_{2}$ and $\Lambda_{3}$, so that observational constraints are relatively insensitive to the choice of $p$ and $q$, and hence to the details of the underlying physics. 


\section{REFERENCES}

[1] A. H. Guth, Phys. Rev. D 23, 347 (1981). For a recent review of inflation and list of references, see A. D. Linde, Particle Physics and Inflationary Cosmology (Harwood Academic, New York, 1990).

[2] A. D. Linde, Phys. Lett. B129 (1993) 177.

[3] J. Ellis, D. Nanopoulos, K. Olive and K. Tamvakis, Phys. Lett. B118 (1982) 335.

[4] M. Grisaru, W. Siegel nd M. Rocek, Nucl. Phys. B159 (1979) 429.

[5] A. D. Linde, Phys. Lett B259, 38 (1991).

[6] A. D. Linde, Phys. Rev. D 49748 (1994).

[7] G. Dvali, Q. Shafi and R. Schaefer, Phys. Rev. Lett. 73 (1994) 1886; L. Randall, M. Soljacic and A. H. Guth, hep-ph/9601296 and Nucl. Phys. B472 (1996) 377.

[8] A. D. Linde and A. Riotto, Report No. FERMILAB-PUB-97-051-A, hep-ph/9703209 (unpublished).

[9] D. H. Lyth, Report No. hep-ph/9609431 (unpublished).

[10] I. Affleck, M. Dine and N. Seiberg, Nucl. Phys. B256 (1985) 557.

[11] For a recent review and a complete list of references, see K. Intriligator and N. Seiberg, Nucl. Phys. Proc. Suppl. 45BC (1996) 1.

[12] J. E. Lidsey, A. R. Liddle, E. W. Kolb, E. J. Copeland, T. Barriero and M. Abney, Rev. Mod. Phys. (1997), Report No. astro-ph/9508078.

[13] A. D. Linde, Phys. Lett. 108B, 389 (1982).

[14] A. Albrecht and P. J. Steinhardt, Phys. Rev. Lett. 48, 1220 (1982).

[15] S. W. Hawking, Phys. Lett. 115B, 295 (1982). 
[16] A. Starobinsky, Phys. Lett. 117B, 175 (1982).

[17] A. Guth and S. Y. Pi, Phys. Rev. Lett. 49, 1110 (1982).

[18] J. M. Bardeen, P. J. Steinhardt and M. S. Turner, Phys. Rev. D 28, 679 (1983).

[19] V. F. Mukhanov, JETP Lett. 41, 493 (1985).

[20] V. F. Mukhanov, Sov. Phys. JETP 67, 1297 (1988).

[21] V. F. Mukhanov, H. A. Feldman, and R. H. Brandenberger, Phys. Rep. 215, 203 (1992).

[22] E. D. Stewart and D. H. Lyth, Phys. Lett. 302B, 171 (1993).

[23] E. F. Bunn and M. White, Astrophys. J. 480, 6 (1996). (Report No. astro-ph/9607060.)

[24] A. Berera, L.-Z. Fang and G. Hinshaw, Report No. astro-ph/9703020 (unpublished). 